\title{
Three Axis Modern Pneumatic Trailer
}

\author{
Sarafaraj J. Mulani ${ }^{1}$, Kunal B. Ramgude ${ }^{2}$, Nishant S. Chothe ${ }^{3}$, Sumit H. Pawar ${ }^{4}$, Shital V. Bhosale ${ }^{5}$ \\ Assistant Professor, Mechanical Engineering Department, AGTI'S DACOE, Karad, Maharashtra, India ${ }^{1}$ \\ Graduate Student, Mechanical Engineering Department, AGTI'S DACOE, Karad, Maharashtra, India ${ }^{2,3,4,5}$
}

\begin{abstract}
Trailer has lots of applications in today's world. In industrial and domestic considerations, tippers can pull a variety of products including gravel, grain, sand, fertilizer, heavy rocks, etc. By considering wide scope of the topic, it is necessary to do study and research on the topic of tipper mechanism in order to make it more economical and efficient. In existing system, tipper can unload only in one side by using pneumatic jack or conveyor mechanism. By this research it is easy for the driver to unload the trailer and also it reduces time and fuel consumption. For making tipper mechanism with such above conditions hydraulic jack mechanism can be used. This paper has mainly focused on above difficulty. Hence a prototype of suitable arrangement has been designed. The vehicles can be unloaded from the trailer in three axes without application of any impact force. The Direction control valves which activate the ram of the hydraulic cylinder which lifting the trailer cabin in require side. By this research it is easy for the driver to unload the trailer and it reduces the time.
\end{abstract}

Keywords: Pneumatic system, valves, trailer.

\section{INTRODUCTION}

Material handling in construction and civil works is one of Automation plays an important role in automobile. the basic necessities. The material supply to civil and Nowadays almost all the automobile vehicle is being construction is provided through trucks, dumper etc. The atomized in order to product the human being. The material should be properly loaded, managed, stacked, automobile vehicle is being atomized for the following transported and unloaded. The dumper carries the material reasons.

which is loaded from the site, where the material is - To achieve high safety initially stored. It is then loaded to the dumper and - To reduce man power transported to the required site and then unloaded. The - To increase the efficiency of the vehicle major issues raises over here, the incompatibility of the site with the fully loaded dumper causes a lot of settling time for the trolley to get the material properly arranged and transportation time to reach its location. The dumper unloads the material in only one direction.

But this incapability can be full new method mechanism as the 'Three Axis Modern Pneumatic Trailer'. Gothic mechanism is an approach to reduce the idle time to settle the dumper. The material is unloaded in any direction and hence can be boldly stated as 'three axis modern pneumatic trailer'[4].The major outcomes of 'three axis modern pneumatic trailer'. Has overcome space requirement which often result in road blocking. hence, we have inversion in the existing mechanism providing the unloading in 180 rotations. this mechanism prevents blocking of road, saves time and enhances productivity at lowest cost. the automotive sector is fast booming section in India. there are variable in automotive industry light and heavy motor vehicle. heavy duty vehicle support as the backbone and confront to the working. a dumper whose material can easily be unloaded in one direction that is mostly to its rear end. these inefficiency is been overcomes three axis modern pneumatic trailer. Automation can achieved through computers, hydraulics, pneumatics, robotics, etc., of these sources, pneumatics form an attractive medium for low cost automation [5].

- To reduce the work load

- To reduce the fatigue of workers

- To high responsibility

- Less Maintenance cost

\section{BASIC IDEA}

This idea was came from the visited a constructional site few days ago. There we found that a dumper was unloading loose material such as sand, gravel, and dirt. A trailer is an integral part of any construction work and hence its role is important for completion of work on site. Typical trailer trucks can generally unload material only exactly of its back side. One thing was remarkable that on complicated locations such as on angular sides and directional sides (left and right) of dumper the unloading of material became quite difficult. In suchconditions dumper truck remained ideal. It consumed extra. Trailers are also the most common cause of accidents involving construction site and plant also. A typical dump truck is equipped with a pneumatically operated open box dead hinged at rear. The front of which can be lifted up to allow the contents to be deposited on the ground behind the truck at side of delivery. Nowadays dumpers with swivel skips could be rotated to sideways (3 directional trailer) which become popular, especially for working in narrow 
IARJSET

sites such as road works. But still this technology is considerations, tippers can haul a variety of products insufficient for fulfilling our requirement for complete including gravel, potatoes, grain, sand, compost, heavy unload. This technology concerns only on solving the rocks, etc. By considering wide scope of the topic, it is problem of unloading on directional sides of trailer. One necessary to do study and research on the topic of tipper of the problems is cited with dumper in the time \& energy mechanism in order to make it more economical and for operating the huge dumper in the proper direction to dump the material carrying in it hence the need of project work riser was is about Three Axis Modern Pneumatic Trailer which dump the material in any direction with moving trolley in any direction. With rise of chances in technology, it is become essential to find a viable alternative to 3 way trailer system.

\section{WORKING PRINCIPLE}

The material is unloading process is done in three axis with the help of locking arrangement and pneumatic system (see Figure.1.). The compressed air is going to the pneumatic cylinder through the direction control valve. The direction control valve is used to control the flow direction of the pneumatic cylinder in both the direction. The pneumatic cylinder is mounted in the rotating table with locking arrangement.

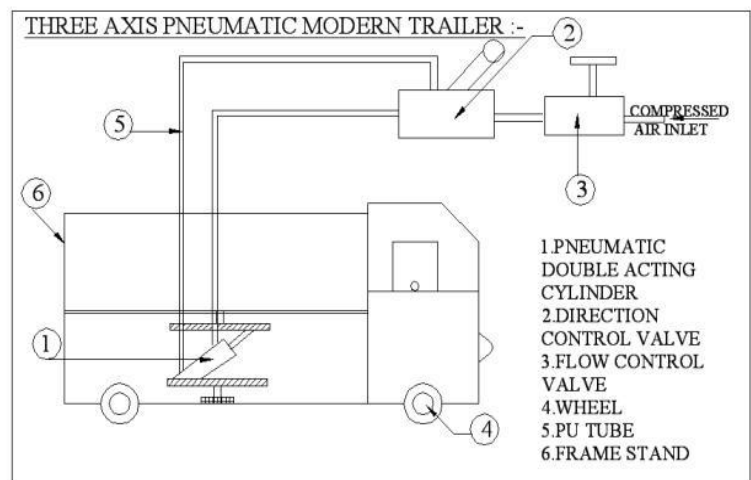

Fig. 1. General layout of tempt

\section{LITERATURE REVIEW}

Ganesh Shindeet al [1] studied the „Modern 3 Ways dropping dumper ${ }^{\text {ee }}$ which has been conceived by observing the difficulty in unloading the materials. The survey in this regards in several automobile garages, revealed the facts that mostly some difficult methods were adopted in unloading the materials from the trailer. They have mainly focused on above difficulty. Hence a prototype of suitable arrangement has been designed. The vehicles can be unloaded from the trailer in three axes without application of any impact force. The Direction control valve which activates the ram of the hydraulic cylinder which lifting the trailer cabin in require side. Further modifications and working limitations will put this work in the main league of use. This concept saves time \& energy which leads to efficient working.

Amboji S. R. et al [2] studied that Tipper has lots of applications in today's world. In industrial and domestic efficient. In existing system, tipper can unload only in one side by using hydraulic jack or conveyor mechanism. By this research it is easy for the driver to unload the trailer and also it reduces time and fuel consumption. For making tipper mechanism with such above conditions both mechanisms namely hydraulic jack and conveyor mechanism can be used. But eventually it comes with question that how both systems can arrange in single set up? Answer to this question is nothing but this research work.

Alley \& McLellan[3] of Glasgow studied hydraulics was being incorporated into truck mounted dump bodies relatively early on, in which record shows one of the first hydraulic dump bodies was the Robertson Steam Wagon with a hydraulic hoist that received power from the trucks engine or an independent steam engine was developed another early hydraulic dump body in 1907 that was power-driven by steam.

\section{BACKGROUND}

Fluid power system is a power transmission system in which the transmission of power takes place through a fluid medium. Such a system avoids the mechanical linkages such as gears, belts, ropes, chains etc. to a great extent of a conventional power transmission system. The transmission of power by fluid power system (see Fig.2.) is most convenient and highly efficient. Due to this, the present conventional transmission systems are being replaced and changed over to fluid power based systems to a pump or compressor which is used to pressurize a fluid. The mechanical energy supplied by the prime mover is converted into the pressure energy by the pump and it is stored in a fluid.

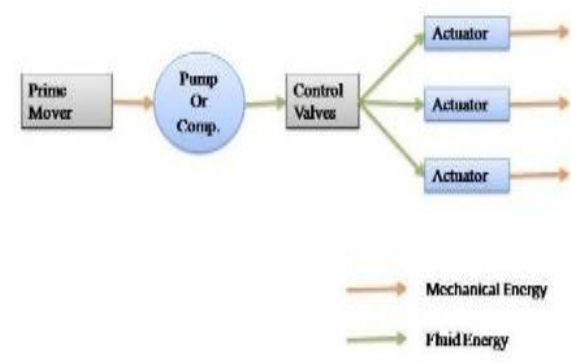

Fig.2. Schematic layout of a fluid power system

The pressurized fluid is now transmitted to different parts of the system through special piping's or tubing's. The various parameters such as pressure and flow rate of the fluid can be controlled by using various control valves. At the desired places of use, the fluid energy is converted 
IARJSET

International Advanced Research Journal in Science, Engineering and Technology

National Conference on Design, Manufacturing, Energy \& Thermal Engineering (NCDMETE-2017)

AGTI's Dr. Daulatrao Aher College Engineering, Vidyanagar Extension, Karad

Vol. 4, Special Issue 1, January 2017

back into mechanical energy by the devices called 1. Design Parts

actuators consisting of cylinders, motors etc. Thus the 2. Parts to be purchased

energy supplied by the prime mover has been transmitted

conveniently through fluid medium to various places and at these places, the mechanical energy has been recovered

For design parts, detailed design is done and dimensions back in a more convenient form. Since the power is transmitted through the fluid as a medium, therefore such a system is called as fluid power system.

\section{A. Construction}

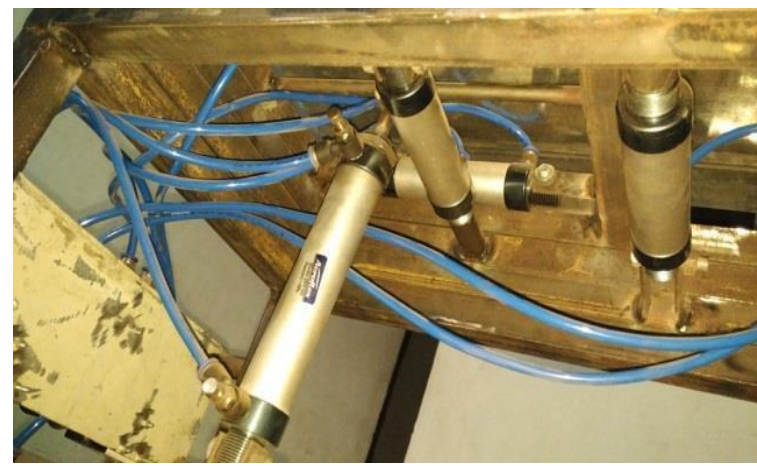

Fig. 3. Constructional details for Pneumatic system

\section{Major Parts:}

The major parts pneumatic three axis modern tipper is described below (Fig. 3.)

- Air compressor

- Direction Control Valve

- Cylinder

- Connecting hoses

- Flow control valve

- Bearing with bearing cap

- Wheel arrangement

- Vehicle model frame

- Rotating Plates

- Lay shaft

- Frame

- Locking system

\section{DESIGN CONSIDERATION}

\section{- INTRODUCTION:}

In our attempt to design a pneumatic robot, we have adopted a very careful approach. Total design work has been divided into two parts mainly,

1. System Design

2. Mechanical Design

System design mainly concern with the various physical concerns and ergonomics, space requirements, arrangements of various components on the main frame of machine, number of controls, positions of this controls, ease of maintenance, scope of further improvements, height of machine components from the ground etc. In mechanical design, the components are categorized into two parts. thus obtained are compared to next highest dimensions which are readily available in the market. This simplifies the assembly as well as post production servicing work. The various tolerances on work pieces are specified in the manufacturing drawing. The process sheets are prepared and passed on to the manufacturing stage. The parts are to be purchased directly are specified and selected from standard catalogues.

\section{- DESIGN OF PNEUMATIC COMPONENTS}

\section{1. vertical piston design:}

For designing the vertical piston, need to apply the clavarinos equation because the material of the piston is ductile material as an aluminium having the tensile stress of $200 \mathrm{~N} / \mathrm{mm} 2$.

Clavarinos equation.

Material of the piston is Aluminium, $\sigma_{\mathrm{t}}=200 \mathrm{~N} / \mathrm{mm}^{2}$

Assume, $p=3 \mathrm{bar}=0.3 \mathrm{~N} / \mathrm{mm}^{2}, \mu=0.36, \mathrm{r}_{\mathrm{i}}=12.5 \mathrm{~mm}$

$\mathrm{t}=0.015 \mathrm{~mm}$

By considering F.O.S.

$1.5 \mathrm{t}=0.015 \times 1.5$

$=0.023 \mathrm{~mm}$

But in market the piston cylinder having minimum thickness of $0.5 \mathrm{~mm}$ is available. So we choose the cylinder having thickness of $0.5 \mathrm{~mm}$.

Available thickness, $\mathbf{t}=\mathbf{0 . 5} \mathbf{m m}$

\section{Piston rod design:}

As the slenderness ratio $(1 / \mathrm{k})$ is less than 40 (As length is very small), we can use the following relations:

$$
d=D \sqrt{\frac{p}{\sigma_{t}}}
$$

$$
\begin{aligned}
& \mathrm{d}=25 \times \sqrt{ } 3 / 200 \\
& \mathrm{~d}=3.06 \mathrm{~mm} \\
& \mathbf{d} \approx \mathbf{5} \mathbf{~ m m} \\
& \text { - Rankine's Formula } \\
& \mathrm{d}=5 \mathrm{~mm} \text { (Dia. of piston rod) }
\end{aligned}
$$

\section{ADVANTAGES AND DISADVANTAGES}

Some of advantages are as follows:

- Lifting cost will be less.

- Free from wear adjustment.

- Less power consumption

- Less skill technicians is sufficient to operate.

- Installation is simplified very much More efficient.

- Power can be easily transmitted 
IARJSET

- Less loss in transmission.

- Very easy holding process

- It requires simple maintenance cares checking and Cleaning are easy because of the main parts are screwed

- Handling is easy

- Repairing is easy.

- Replacement of parts is Easy.

Some of Disadvantages are as follows:

- Less load carrying capacity as compare to hydraulic system.

- Air compressor is required.

- Higher operating cost.

- Compressor required for this system is quite bit noisy.

- Due to sliding motion of parts, more wear \& tear occurs.

\section{APPLICATIONS}

The following applications are considered of material handling processes.

- Packing: packing is that puts goods into boxes to protect them and to make them easier to carry while in transit. In this the required quantity of the material is loaded in trailer and can be unloaded at required direction.

- Material loading: In this, any bulk and unit load can be loaded, transported and unloaded at required place and required direction.

- Dispensing: dispensing is distributed or weighted out in carefully determined portions. In which material is distributed at any three directional places.

- Palletizing: In this the material is filled in pallets and these pallets are carried or supported by trailer and transported to required place by using forklift.

- Material dropdown: the finished products on machine is dropdown by using pneumatic trailer.

- Pick \& place: pick up and placement of all types of materials at required place.

\section{FUTURE SCOPE}

As the world progressing at faster rate we meet mover and mover huge construction which head to be dig big and big amount of the earth and thus more efficiently working equipment are to be required and hence the Development of Three Axis Modern Pneumatic trailer may be used more than the two way or one way. India is progressing at higher rate and hence infrastructural development is on its high. Hence the future of this project work seems promising. The project work can be modified further more on following basis:

- Dual stage cylinders can be used.

- This system can be used by using hydraulic system instead of pneumatic systems for higher loads.

- Wheel steering can be adopted for avoiding the lifting of vehicle along with trailers.
- Sensors and alarms can be used to avoid risks.

- This arrangement can fitted on Automated Guided Vehicles (AGV).

- This system can be used in heavy vehicles like dumpers etc. with the help of hydraulics.

- In an automation industries this system can be used with the help PLC system.

\section{CONCLUSION}

The developed prototype exhibits the expected results. Further modifications and working limitations will put this work in the main league of use. This concept saves time \& energy which leads to efficient working. This further line should be modeled using equations and an experimental agreement. The constructional work or the infrastructural work demands efficient and user friendly machinery which will lead to more and more use of three Axis Moder Pneumatic trailer.

\section{REFERENCES}

[1] Ganesh Shinde, PrachiTawele, LaukikRaut, "Designs and Development of 3-Way Dropping Dumper" International Journal of Emerging Technology \& Advanced Engineering, (ISSN 2250-2459, ISO 9001:2008 Certified Journal, Volume 4, Issue 9, September 2014).

[2] P. Manasa, C. VijayaB. Reddy, "Modeling and Analysis of Tractor trolley Axle using Antsy Design and Fabrication of Unidirectional Dumper", Journal of Mechanical and Civil, E-ISSN-2278-1684ISSN-2320-334X, Volume Vol. 6, Issue 5 (MAY-JUNE) PP 88-92.

[3] Wood, Donald, Dump Trucks [3]. 729 Prospect Ave. Osceola, WI 54020: MBI Publishing Company. pp. 6-9. Wood, Donald (2001). Dump Trucks. 729 Prospect Ave. Osceola, WI 54020: MBI Publishing Company. pp. 11-3.

[4] AmbojiS. R., Humane Y. A.,ChavanR.R., PatilJ. C, KshirsagarP. R., "Design and fabrication of 3 way tipper mechanism", International journal of researching advanced technology vol, No.4, April 2014 E ISSN 2321-9637.

[5] Padmanabhan. S., Chandrasekaran.M.and Srinivasa Raman. V., "Design Optimization of Worm Gear drive", International Journal of Mining, Metallurgy \& Mechanical Engineering, Vol. 1, Issue 1 (2013) ISSN 2320-4060. 